\title{
Estudo Prospectivo Exploratório das Patentes de Métodos de Aprendizagem de Máquina Aplicados ao Mercado Financeiro
}

\author{
Exploratory Prospective Study of Methods of Machine Learning Patents \\ Applied to the Financial Market
}

\author{
Vitor M. Quintella ${ }^{1}$ \\ Cristina M. Quintella ${ }^{1}$ \\ Antônio Francisco A. Silva Junior ${ }^{1}$ \\ Cristiano de Oliveira Hora Fontes ${ }^{1}$ \\ ${ }^{1}$ Universidade Federal da Bahia, Salvador, BA, Brasil
}

\begin{abstract}
Resumo
Neste trabalho é realizada uma prospecção tecnológica do uso da tecnologia de aprendizagem de máquina aplicada à negociação e ao investimento financeiro. A rápida expansão das aplicações de Tecnologia da Informação (TI) no campo das finanças tem gerado uma série de mudanças nas suas práticas impactando a vida de investidores e de todos os cidadãos. Foi utilizada base de dados de documentos de patentes da Espacenet, o escopo de pesquisa permitiu a análise de 257 patentes. As patentes analisadas no escopo são datadas de 1991 a 2018. O software Questel Orbit ${ }^{\circledR}$ foi utilizado para análise dos dados. As principais aplicações são orientadas para a identificação de outliers e a realização de feature selection e clustering. Não foi identificada empresa que tenha como atividadefim atuar no mercado financeiro, indicando a existência de estratégias de manutenção de segredo de negócio. Foi verificado o rápido crescimento do mercado e a possibilidade de amadurecimento da tecnologia diante do cenário contemporâneo.
\end{abstract}

Palavras-chave: Aprendizagem de Máquina. Mercado Financeiro. Investimento. Negociação. Prospecção.

\begin{abstract}
This is a prospective study of the uses of machine learning technology applied to financial market trading and investment. Quick expansion on TI technology generated effective changes on daily financial routine. As futher changes may be expected, this prospection analyzed 257 patents from Espacenet database processed via Questel Orbit ${ }^{\circledR}$ software. It was found in 2016 a peak in 1 st patent deposits that remains until the present. Most of applications was on outliers, feature selection and clustering. No holder was identified with financial core activity, pointing out the possibility of trade secret strategies. China and USA were the biggest depositors and the biggest market of these applications. The outstanding growth of the market and the identification of a horizon of technological maturation processes is a consequence of the recent development in process power, data readiness and cloud-based technology.
\end{abstract}

Keywords: Machine Learning. Financial Market. Investment. Trading. Prospection.

Área Tecnológica: Engenharia de Produção. 


\section{Introdução}

Os mercados financeiros são parte significativa do cotidiano da sociedade moderna e inevitavelmente influenciam a curto, médio e longo prazo o dia a dia dos cidadãos contemporâneos. Sua maior e mais evidente influência é sobre a economia.

Conforme alegam Chakraborty e Joseph (2017), duas recentes tendências influenciam no desenvolvimento de metodologias financeiras. Primeiro, o aumento da capacidade de processamento que tem permitido ao machine learning alcançar novos patamares de aplicação. Segundo, o aumento da disponibilidade de dados, alcançado no geral de diversos conjuntos de big data. Ambos têm atuado de forma complementar, pois a maior capacidade de processamento permite trabalhar a maior quantidade de dados, e a maior quantidade e diversidade de dados permite o desenvolvimento de técnicas mais complexas e completas.

Das diversas aplicações de aprendizagem de máquina em finanças, duas se relacionam especialmente ao campo do mercado financeiro: aquelas orientadas para a gestão de portfólios e de investimentos e aquelas relacionadas para a melhor valoração de valores presentes $e$ futuros de produtos (e.g. ações de empresas, commodities, moedas e derivativos). Essas duas possuem tipicamente o alto volume e a diversidade de informações, ambos necessários para caracterizá-los como big data, e possuem uma velocidade de atualização de informações que justifica a utilização de machine learning, especialmente para analises dinâmicas. Essas duas áreas são dependentes, já que uma trata do entendimento do objeto de negociação em si e a outra trata da gestão e do processo de tomada de decisão sobre a negociação dos objetos.

Quanto ao conceito de machine learning, é importante destacar que ele se diferencia do conceito de inteligência artificial, embora muitas vezes sejam utilizados indistintamente. Khargonekar e Dahleh (2018) descrevem que o termo "artificial inteligence" foi cunhado por John McCarthy em 1956 e representa um largo conceito de máquinas capazes de realizar tarefas que são compreendidas como inteligentes (e.g. ou autônoma e adaptiva) enquanto o termo machine learning foi cunhado pouco depois por Arthur Samuel em 1959 e representa a capacidade de um programa aprender uma determinada tarefa com base em determinada experiência. Assim, o conceito de machine learning pode ser compreendido como uma especificação do conceito de inteligência artificial e é, na prática, uma forma prática de tratar volumes de informações extensas, contínuas e mutantes sem a necessidade de um trabalho infindável de um analista financeiro (no caso da nossa aplicação).

Embora muitas pesquisas se desenvolvam na área, alguns métodos prevalecem como fundamentos para a aplicação de machine learning ao mercado financeiro. $\mathrm{O}$ trabalho de Cavalcante et al. (2016) mapeia as principais pesquisas na área, seus autores, objetivos e métodos. Os seus objetivos tipicamente podem ser: clustering, de-noising, feature selection, forecasting, outlier detection e segmentation. Quanto às suas aplicações, três são as principais categorias trabalhadas: o pré-processamento, a previsão e o trading. O pré-processamento inclui métodos como seleção e extração de variáveis, filtragem de ruídos e retirada de outliers, segmentação de séries temporais e agrupamento (clustering). A previsão inclui métodos como redes neurais artificiais, vetores de suporte, métodos híbridos, métodos de otimização e modelos combinados. Os dois 
possuem abordagens distintas, mas complementares. Enquanto o pré-processamento prepara as séries temporais para uma melhor interpretação, a previsão realiza o processo de buscar a melhor aderência da expectativa com o futuro. $\mathrm{O}$ trading define estratégias de compra e venda e de avaliação de retornos, desenvolvendo algoritmos que continuamente tomam posições de mercado efetuando transações em busca de resultados financeiros.

Por fim, Cavalvante et al. classificam as pesquisas referentes aos seus métodos e tais métodos são utilizados neste paper para definir o escopo do levantamento de patentes registradas. Os métodos em questão são: dynamic time wrapping, f-score or f-measure, fuzzy clustering, independent components analysis, $k$-means, $k$-nearest neighbor, linear discriminant analysis, local linear embedding, multilayer perceptron, perceptually important points, principal components analysis, self organizing map, suport vector machine, turning points e vonoroi diagram.

No caso de os desenvolvedores quererem apropriar suas tecnologias, desse modo controlando quem comercializa e quem produz e usando seus desenvolvimentos, torna-se essencial apropriar-se por meio de propriedade industrial. Podem ser utilizadas diversas formas como patentes de invenção focadas em métodos e processos, e registros de software. Podem ainda ser realizadas apropriações complementares como desenhos industriais e marcas. Registros de software têm dificuldades de serem comprovados judicialmente já que, de fato, o código fonte é pelo menos parcialmente cópia de um anteriormente registrado. Adicionalmente, existem diversos modos de obter o mesmo resultado usando diversas variações de códigos. Assim, o formato de patente é a melhor forma de comprovar a autoria e a titularidade.

De tal forma, este trabalho pretende realizar uma prospecção patentária exploratória da aplicação de machine learning a mercados financeiros. $\mathrm{O}$ trabalho almeja não apenas levantar as patentes que foram registradas no tema, mas, também, estudar o processo de amadurecimento e de desenvolvimento dessas patentes, identificando - inclusive - os métodos proeminentes e as organizações com maior presença. Por fim, o trabalho realizado, servirá para estruturar e orientar pesquisas futuras, estabelecendo a situação de apropriação patentária próprias delas.

\section{Metodologia}

Foram utilizados a base de dados de documentos de patentes da Espacenet e o software Questel Orbit ${ }^{\circledR}$, em seguida, foram mapeadas as famílias de patentes. Doravante será usada a palavra "patentes" para indicar documentos de patentes, independentemente do seu status legal. O escopo foi estudado num longo período de tempo, e os dados aqui apresentados foram baixados em $1^{\circ}$ de julho de 2018 .

As buscas foram feitas tanto em conceitos criados pela plataforma Orbit ${ }^{\circledR}$, como em radiais de palavras-chave, incluindo tempos verbais e variações de número, nas reivindicações $e$ no título de cada patente. Ambas as buscas foram ajuntadas de modo a se conseguir o maior número de patentes. 
Na Tabela 1 estão demonstradas algumas das opções de busca testadas.

Tabela 1 - Escopo

\begin{tabular}{|c|c|c|c|c|c|c|c|}
\hline $\begin{array}{l}\text { APRENDIZAGEM } \\
\text { DE MÁQUINA }\end{array}$ & $\begin{array}{c}\text { TÉCNICAS DE } \\
\text { APRENDIZAGEM DE } \\
\text { MÁQUINA* }\end{array}$ & $\begin{array}{l}\text { G06Q- } \\
\text { 040/00 }\end{array}$ & $\begin{array}{l}\text { G06Q- } \\
\text { 040/02 }\end{array}$ & $\begin{array}{l}\text { G06Q- } \\
\text { 040/04 }\end{array}$ & $\begin{array}{l}\text { G06Q- } \\
040 / 06\end{array}$ & $\begin{array}{l}\text { G06Q- } \\
\text { 040/08 }\end{array}$ & $\begin{array}{l}\text { FAMílIAS DE } \\
\text { PATENTES }\end{array}$ \\
\hline \multirow[t]{2}{*}{$X$} & & & & & & & 46.201 \\
\hline & $\mathrm{X}$ & & & & & & 82.267 \\
\hline \multirow[t]{7}{*}{ OR } & OR & & & & & & 82.267 \\
\hline & & $\mathrm{X}$ & & & & & 50.265 \\
\hline & & & $X$ & & & & 27.937 \\
\hline & & & & $X$ & & & 12.596 \\
\hline & & & & & $\mathrm{X}$ & & 5.773 \\
\hline & & & & & & $X$ & 5.522 \\
\hline & & & & OR & OR & & 16.142 \\
\hline OR & OR & & & OR & OR & & 257 \\
\hline
\end{tabular}

*De acordo com Cavalcante et al. (2016)

Fonte: Elaborada pelos autores deste artigo (2018)

Para Aprendizado de Máquina foram usados: machine learning or support vector machine or machine learning system or machine learning model or machine learning classifier or machine learning training or trained machine learning

No entanto, esse escopo mostrou que precisaria ser mais robusto, já que Aprendizado de Máquina é ainda uma tecnologia muito recentemente disseminada e não tem denominações universalmente consensuadas. Para solucionar esse problema, foram mapeadas as técnicas computacionais usualmente empregadas, com base em Cavalcante et al. (2016), sendo pesquisados os conceitos e os radicais de: (self organizing map) or (suport vector machine) or (vonoroi diagram) or (f-score or f-measure) or (multilayer perceptron) or (turning points) or (perceptually important points) or (principal components analysis) or (independent components analysis) or (linear discriminant analysis) or ( $k$-means) or (local linear embedding) or ( $k$-nearest neighbor) or (fuzzy clustering) or (dynamic time wrapping) or (neural network) or (text mining)

Os códigos de classificação patentária internacional (IPC) utilizados foram criados em 2012, devido à novidade desse tipo de tecnologias, e permitiram restringir o foco da busca em métodos de processamento de dados para finanças com foco em:

a) Negociação, por exemplo, ações, commodities, derivativos ou câmbio de moedas (G06Q 40/04).

b)Investimento, por exemplo, instrumentos financeiros, gestão de carteiras ou gestão de fundos (G06Q 40/06).

A classificação G06Q 40/00 (Finança; Seguro; Estratégias tributárias; Processamento de impostos corporativos ou de renda) não foi utilizada por ser genérica e não focar no assunto deste trabalho. 
As classificações que fugiam do escopo deste trabalho e, por isso, não foram utilizadas são: G06Q 40/02 (Banca, e. cálculo de juros, aprovação de crédito, hipotecas, home banking ou banco on-line) e G06Q 40/08 (Seguros, por exemplo, análise de risco ou pensões).

As patentes foram analisadas para retirar as duplicidades e as patentes fora do foco deste trabalho, foram processadas 257 famílias de patentes no total.

\section{Resultados e Discussão}

A Figura 1A mostra o status legal das patentes. Pode-se observar que o percentual de patentes "vivas", ou seja, patentes que aguardam exame $(65,8 \%)$ e patentes concedidas $(17,5 \%)$, é bastante alto $(83 \%)$ em relação a outras tecnologias, o que é causado pelo alto número de patentes ainda não examinadas, o que leva a crer que essa tecnologia é realmente emergente.

É demonstrado na Figura 1B a evolução anual de primeira prioridade das famílias de patentes, o que confirma que a maioria das patentes é relativamente recente.

Figura 1 - (A) Status legal das patentes; (B) Evolução anual do número de famílias de patentes, em relação ao ano de primeira prioridade

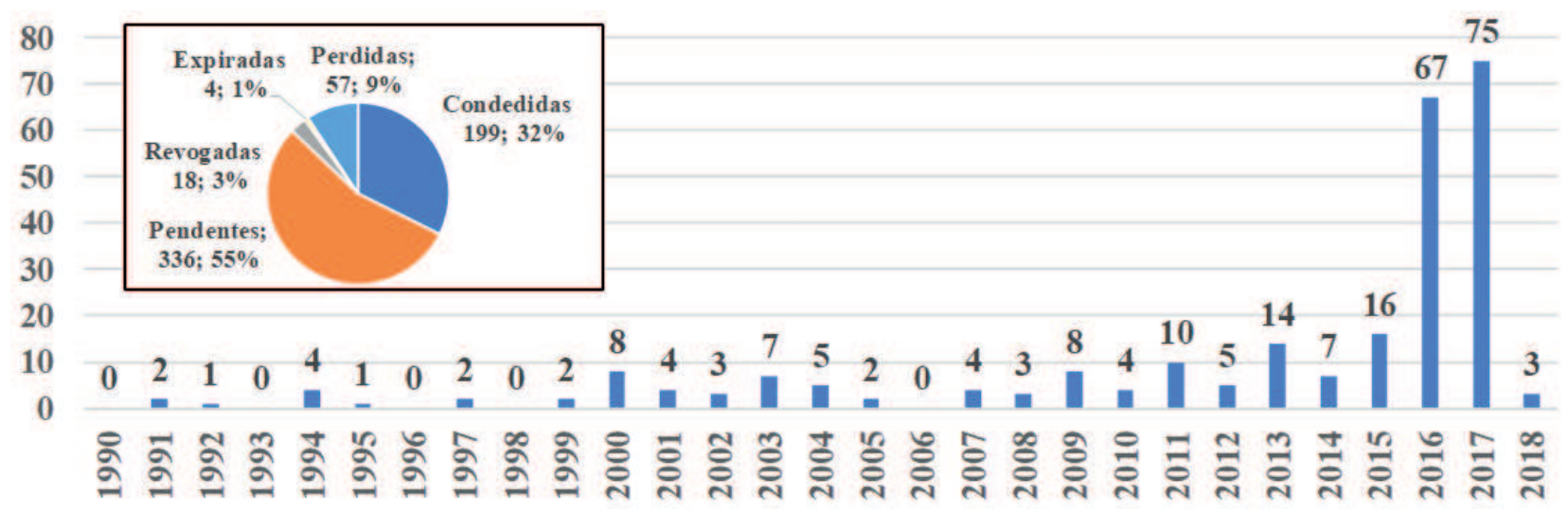

Fonte: Elaborada pelos autores deste artigo (2018)

Pode-se observar que, desde 1991 até 2014, existem alguns depósitos anuais que flutuam ao longo do período, sem um padrão estabelecido. Essas patentes são atribuídas a entrantes iniciais neste campo tecnológico, mas ainda sem volume de apropriação que permita de fato produzir e comercializar as tecnologias de modo amplo.

O máximo em 2000 é causado por quatro famílias de patentes de titularidade Nyse American que se referem sistemas e métodos para negociar fundos ativamente gerenciados, carteiras de modelagem, avaliação da integridade de carteiras e cobertura cambial negociada entre fundos.

Como este trabalho utilizou um grupo de patentes identificado em $1^{\circ}$ de julho de 2018 , e devido aos 18 meses de sigilo, os anos de 2017 e de 2018 ainda não têm o total de patentes, portanto, seus números devem ser desconsiderados.

Observa-se que a maioria das patentes está em 2016 e 2017, confirmando que essa é uma tecnologia muito recente. 
A Figura 2 mostra o Mapa-Múndi com a distribuição patentária, sem considerar protocolos que ainda não tenham indicado os países como os da Comunidade Europeia (EP) e os do Tratado de Cooperação Patentária (PCT).

A Figura 2A mostra os países de primeira prioridade. É possível observar que os países que se destacam no desenvolvimento da tecnologia são China (48\%), Estados Unidos (33\%) e Japão (12\%). Além desses, algumas outras patentes são Grã-Bretanha, Coreia, Taiwan, Austrália, Canadá e Índia.

São demonstrados na Figura 2B os países nos quais estão sendo publicadas as solicitações de patentes. É possível ver que os países são bem semelhantes aos de prioridade. Devido ao período de sigilo, que impede publicação das patentes, e por que patentes via PCT podem esperar até 36 meses para que sejam definidos os países que serão requeridos, é possível que de fato haja muitos outros países onde já foram requeridas patentes. Assim, os países de publicação com maior número de patentes são os mesmos dos de primeira prioridade: China (51\%), Estados Unidos (35\%) e Japão (16\%).

No entanto, pode-se observar que alguns países que não produzem a tecnologia estão publicando as patentes, sendo os mercados potenciais para exportação da tecnologia: Senegal, Hong Kong, México, Argentina, Brasil, Itália e Polônia.

Figura 2 - Mapa-Múndi com a distribuição patentária, sem EP e PCT: (A) Países de primeira prioridade; (B) Países de publicação

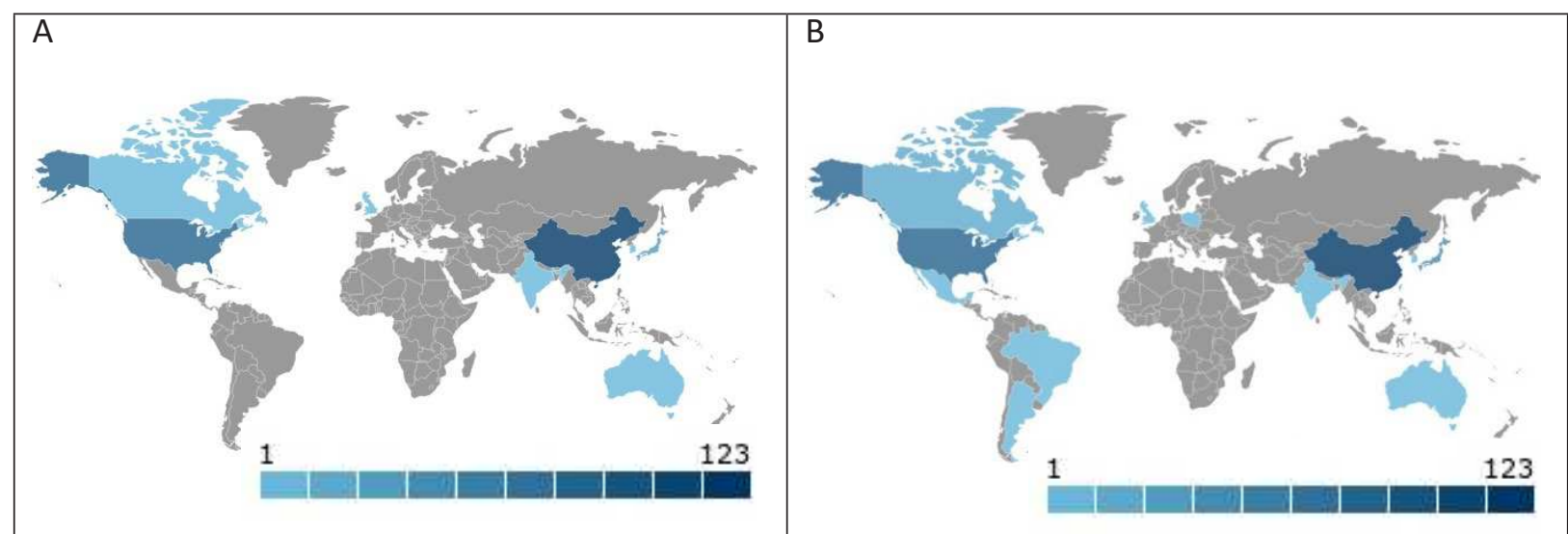

Fonte: Elaborada pelos autores deste artigo (2018)

A análise dos IPCs obtidos permitiu ver que, associados aos dois IPCs escolhidos no escopo deste trabalho, apareceram outros usos do Aprendizado de Máquina. A previsão ou otimização, por exemplo, programação linear, "problema de vendedor ambulante" ou "problema de corte de estoque" (G06Q-010/04) está presente em 52 patentes. Os recursos, fluxos de trabalho, gestão humana ou de projeto, por exemplo, organizar, planejar, programar ou alocar tempo, recursos humanos ou de máquinas; planejamento empresarial; modelos organizacionais (G06Q010/06), têm 37 patentes. O marketing, por exemplo, pesquisa de mercado e análise, topografia, promoções, publicidade, perfis de compradores, gerenciamento de clientes ou recompensas; estimativa ou determinação de preço (G06Q-030/02), tem 37 patentes. 
Os domínios tecnológicos das patentes mostraram que estão concentrados naqueles tradicionais de Tecnologia da Informação e Comunicação (TIC). métodos de TI para gestão têm $100 \%$ das patentes, tecnologia de computadores tem $5,1 \%$ e comunicação digital tem $4,7 \%$. No entanto, o Aprendizado de Máquina está sendo já utilizado em patentes para outros campos tecnológicos: maquinaria elétrica, aparelhos, energia; controle; tecnologia audiovisual; telecomunicações; medição; ferramentas da máquina; outras máquinas especiais; e máquinas têxteis e de papel.

Inicialmente foram feitas buscas pelos usos mais comuns de Aprendizado de Máquina. As buscas foram feitas tanto em conceitos criados pela plataforma Orbit ${ }^{\circledR}$ como em radiais de palavras-chave, incluindo tempos verbais e variações de número, nas reivindicações e no título de cada patente. Ambas as buscas foram ajuntadas de modo a se conseguir o maior número de patentes. Foram utilizados:

a) Remoção de ruído: removing noise or denoising or noise removal processing or de noise or signal denoising or denoised image or noising threshold.

b) Outliers: outlier detection or outlier or training data or anomaly detection or outlier data or removing outlier or abnormal value or outlier identification or hierarchic clustering or mahalanobis distance or random forest or statistical outlier or abnormal point or outlier analysis.

c) Agrupamentos: cluster or clustering or cluster center or euclidean distance or hierarchic clustering or cluster analysis or clustering result or $k$ means clustering or initial cluster or clustering module or clustering unit or data clustering or clustering data or k cluster or clustering step.

d) Segmentação: segmentation or segmentation module or segmentation result or segmentation processing or segmentation unit or segmentation threshold or segmentation accuracy or automatic segmentation or accurate segmentation or segmentation step.

e) Feature selection: feature selection or feature extraction or feature vector or support vector machine or dimensionality reduction or feature selection module or feature subset or optimal feature subset or candidate feature or redundant feature or ranking feature or optimal feature

Está representado na Tabela 2 o número de patentes para cada uso do Aprendizado de Máquina. É possível observar que a busca de remoção de ruído identificou apenas uma patente, o que mostra que os redatores de patentes não estão incluindo esse aspecto no título, nem das reivindicações. Feature selection obteve perto de $22 \%$ sendo bastante utilizado. Outliers e Agrupamentos aparecem com maior regularidade aprentando ser a denominação mais usual, com praticamente $30 \%$. No entanto, observa-se que a busca pelos usos A, B, C, D e E ainda não incluiu 58\% das patentes, mostrando que, na sua maioria, uma série de outras denominações de usos são utilizadas. O que já era esperado, comprovando que, sendo uma tecnologia muito recente, ainda não há consenso de denominações, justificando, assim, o escopo mais robusto utilizado para este trabalho. 
Tabela 2 - Número de famílias de patentes para diversos tipos de tratamentos matemáticos: Remoção de ruído (A); Outliers (B); Agrupamentos (C); Segmentação (D); Feature selection (E); Outros diferentes destes (F)

\begin{tabular}{cccc} 
Item & Tipo de tratamento & $\begin{array}{c}\text { Número Famílias de } \\
\text { Patentes }\end{array}$ & $\begin{array}{c}\text { Percentual de Famílias } \\
\text { De Patentes }\end{array}$ \\
A & Remoção de ruído & 1 & $0,39 \%$ \\
B & Outliers & 75 & $29 \%$ \\
C & Agrupamentos & 70 & $27 \%$ \\
D & Segmentação & 25 & $9,7 \%$ \\
E & Feature selection & 56 & $22 \%$ \\
F & Outros diferentes destes & 149 & $57,9 \%$ \\
\hline
\end{tabular}

Fonte: Elaborada pelos autores deste artigo (2018)

A tecnologia de Aprendizagem de Máquina, apesar de relativamente recente, tem 99 titulares, sendo $81 \%$ de empresas e apenas $17 \%$ de academias. O setor acadêmico compreende 17 universidades chinesas, uma de Taiwan e outra dos Estados Unidos.

Há apenas uma patente em nome de pessoa física, e o titular é Scott Eder Jeffrey (JEFFREY, 2016), que foi transferida para a empresa Asset Reliance em 15 de maio de 2016. Esse inventor tem uma série de outras patentes que estão apropriadas ou licenciadas em nome da empresa Asset Trust, trata-se de uma companhia parente da empresa General Electric.

As empresas que têm maior portfólio patentário são Alibaba Holding, Eder Jeffrey, Fujitsu, Nyse American, Thomson Reuters Global Resources Unlimited, Beijing Beta Zhitou Technology, Beijing Bita Zhizhi Technology, Locus, Nippon Telegraph \& Telephone, Tsinghua University, Yahoo Japan, e Asset Trust.

Está representada na Figura 3 a evolução das aplicações ao longo do tempo pelos 10 maiores titulares, usando o ano de primeira prioridade, destacando a estratégia de patentes e identificando novos entrantes ou titulares que não estão mais envolvidos nesta tecnologia.

Observa-se que o titular mais antigo é Nippon Telegraph \& Telephone, que protocolou patentes apenas no Japão nos anos de 1996 e 1997. Tem portfólio, em média, de 22 anos com três famílias de patentes. As patentes em questão consistem em um método de aprendizagem de série temporal destrinchado em três variáveis, sendo que apenas uma das séries trata explicitamente de valor de ações. Embora o foco da empresa não seja finanças, a árvore corporativa dela é complexa, tendo depositado uma média de duas mil patentes ao ano desde 1976. De tal modo, as patentes em questão podem ter surgido como um spinoff de pesquisas de séries temporais naturais do desenvolvimento de tecnologia para sua atividade-fim.

Outro titular antigo é Asset Trust com duas patentes em 1997, depois continuou a atuar em conjunto com o inventor Jeffrey Edider entre 2001 e 2005, e já deixou de patentear em Aprendizado de Máquina. Existe portfólio, em média, de 12 anos com duas famílias de patentes. Essa empesa é uma companhia da General Eletric, responsável pela gestão de fundos dos seus funcionários. A empresa teve um pico de depósito de patentes até 2004, momento no qual ela começou a reduzir seus depósitos até parar completamente o processo.

Jeffrey Eder, que se apropria desde 2004 como titular e/ou inventor, licencia para Asset Reliance, Asset Trust e Valueminer (JEFFREY, 2004a; JEFFREY, 2004b; JEFFREY, 2009a; 
JEFFREY, 2009b; JEFFREY, 2016). Tem portfólio, em média, de 10 anos com seis famílias de patentes. A companhia é familiar de consultoria e investimentos, fazendo parte da empresa Martin Jeffrey e atuando recentemente com hightecs bancárias.

O titular mais antigo com patentes ainda ativas é a Nyse American, que, no entanto, deixou de apropriar nesse escopo recentemente. Tem portfólio, em média, de nove anos com quatro famílias de patentes. A companhia é a antiga American Stock Exchange (AMEX), bolsa de valores de Nova York. A ausência de mais patentes indica a possibilidade de futura expansão do escopo, tendo em vista que é improvável que essa companhia tenha parado o desenvolvimento de tais tecnologias. Uma futura investigação da companhia pode ainda desvendar o surgimento de uma companhia filha que trabalhe especificamente com investimentos.

A Thomson Reuters Global Resources Unlimited iniciou seu portfólio atualmente ativo em 2009. Tem portfólio, em média, de seis anos com quatro famílias de patentes. Consiste em uma subsidiária da Thomson Reuters Corporation, sendo uma companhia de gestão de fundos. O depósito médio anual da companhia é de cinco patentes, atuando majoritariamente na área de tecnologia aplicada à gestão de portfólios.

Figura 3 - Dez maiores titulares: (A) Evolução anual do portfólio de patentes; (B) Idade e número de patentes do portfólio

(A) Evolução anual do portfólio

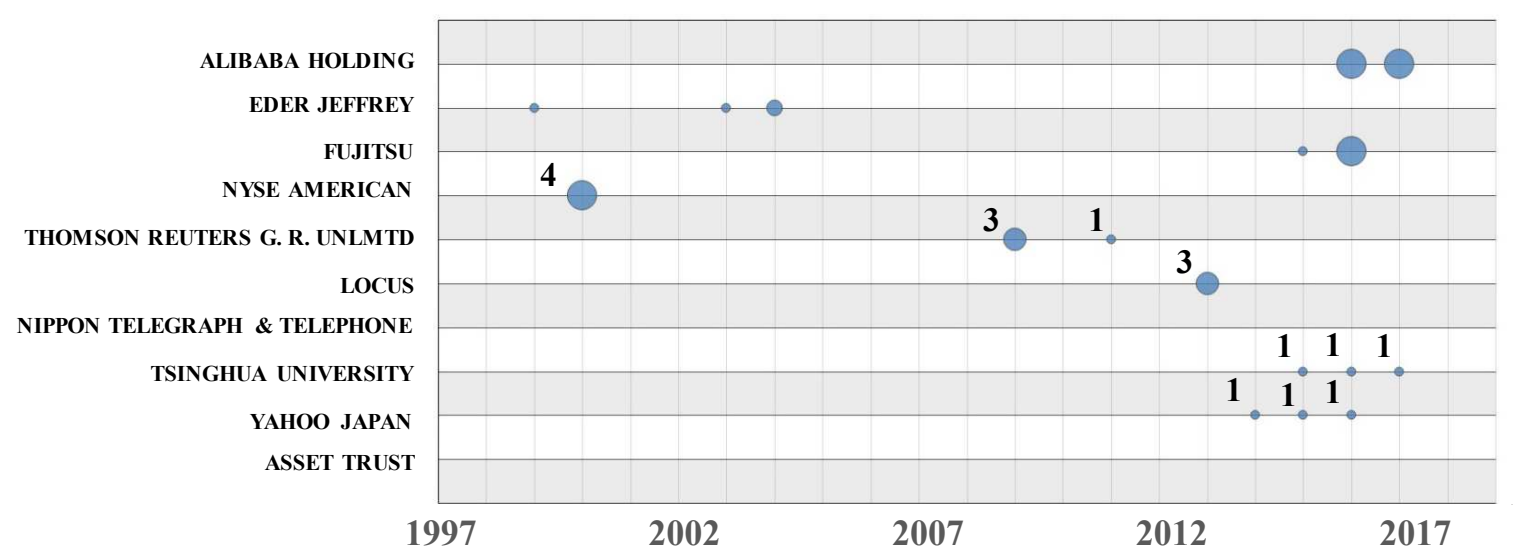

(B) Idade e número de patentes do portfólio

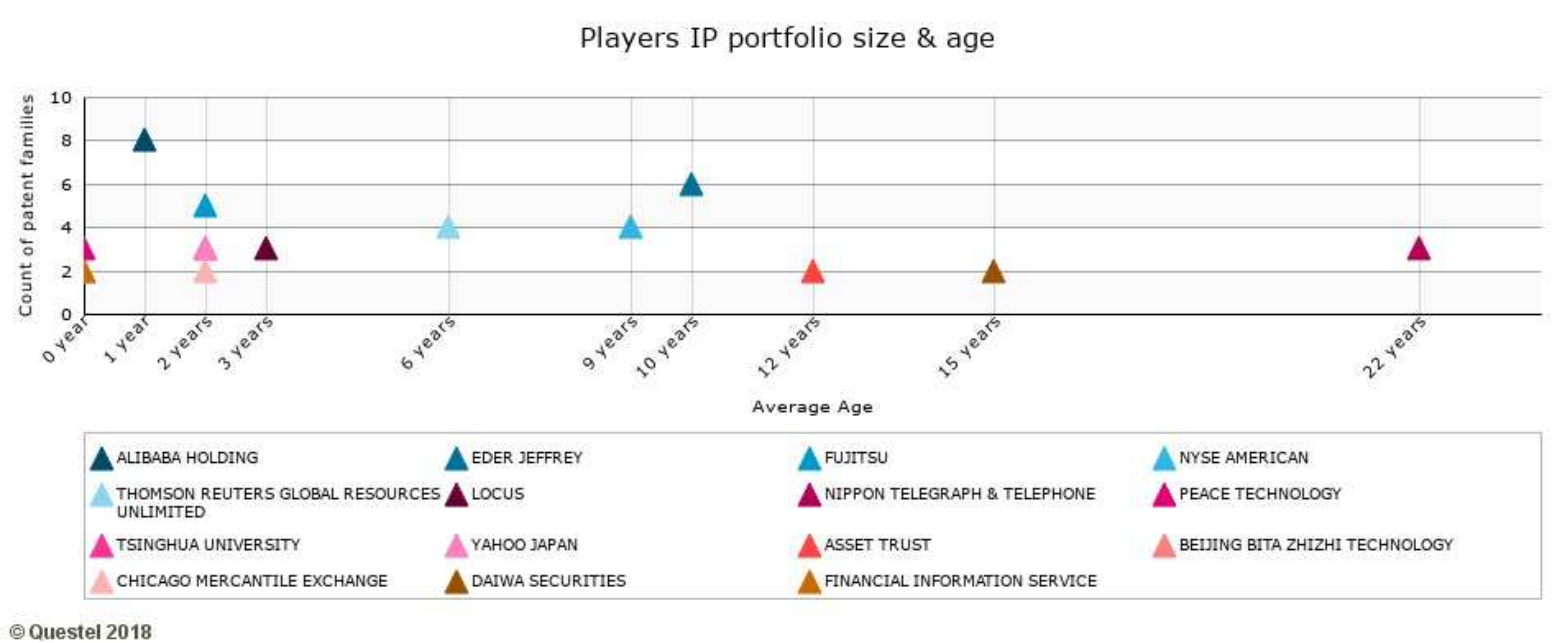

Fonte: Elaborada pelos autores deste artigo (2018) 
A Locus entrou na apropriação dessa tecnologia em 2013. Tem portfólio, em média, de três anos com três famílias de patentes. É uma empresa que atua com equipamentos industriais (trocadores de calor, válvulas e tubulações) e automação, essa empresa pertence à Seven Seas Holding que atua no mercado imobiliário e financeiro. A empresa Locus possui 83 patentes com uma média de quatro patentes publicadas por ano desde 1998.

Tsinghua University e Yahoo Japan são entrantes com portfolio de três famílias de patentes. A Tsinghua University tem portfólio, em média, de um ano com três famílias de patentes. consiste em universidade estatal da china, cujo depósito de patentes anual tem crescido em 200 patentes ao ano, tendo alcançado um depósito de três mil patentes nos últimos anos. O crescimento regular de seus depósitos é consequência das políticas nacionais da China que buscam a presença forte e crescente em propriedade intelectual. A universidade registra patentes nos mais diversos temas, sendo dois dos principais Finanças e Aprendizagem de Máquina. O Yahoo Japan tem portfólio, em média, de três anos com três famílias de patentes, é uma joint venture entre a americana Yahoo (agora Altaba) e a japonesa SoftBank. Embora tenha surgido em 1996, a empresa utilizava as tecnologias já existentes das suas companhias geradoras, tendo começado a depositar tecnologia própria a partir de 2006 e aumentado sua produção continuamente, alcançando hoje a marca de 2.500 patentes.

Fujitsu é um entrante na tecnologia e está ativo, tendo uma patente em 2015 e quatro patentes em 2016. Possui portfólio, em média, de um ano com cinco famílias de patentes. A companhia tem depositado continuamente 3.500 patentes anuais na área de tecnologia da comunicação, atualmente é proprietária de mais de 230 mil patentes. A empresa atua especialmente na área de eletrônicos. Sua área de atuação específica fornece o embasamento necessário para a atuação dentro do escopo em questão.

Alibaba Holding é o mais recente entrante na tecnologia, tendo iniciado seus depósitos em 2016. Tem portfólio, em média, de um ano com oito famílias de patentes. Essa gigante chinesa atua em diversas áreas, como e-commerce, computação nas nuvens, streaming além de atuar como investidora parceira de uma série de companhias da área tecnológica. A chinesa multiplicou sua receita continuamente nos últimos cinco anos, alcançando a marca dos 11 bilhões de dólares em 2017. A sua atuação no escopo dessa pesquisa parece ser uma consequência natural da sua estratégia de expansão de negócios. Porém, destaca-se que a essa empresa restringe sua atuação no mercado chinês, de tal modo que ocasionalmente irá estancar o enorme crescimento que tem alcançado. Tal perspectiva sugere que ela pode continuar um player crescente no escopo da pesquisa nos próximos anos, mas que o crescimento diferenciado irá eventualmente estabilizar.

Percebe-se, ainda, que a existência de players com atividade-fim financeira é praticamente inexistente dentro do escopo avaliado. Duas são as principais possíveis explicações para tal incongruência: ou as empresas que possuem atividade-fim financeira evitam o uso de patentes para resguardar seus segredos de negócio, ou as empresas protegem seus métodos por meio de depósitos de patentes externos ao escopo deste trabalho, evitando o uso de classificadores IPC de finanças. Tais hipóteses podem ser verificadas em futuros estudos que expandam o escopo ou que atuem rastreando as patentes de companhias que atuam na área.

O titular mais citado é Jeffrey Edider, ele é um inventor também muito citado. No entanto, o inventor mais citado é, de longe, Jason Crabtree, cujos titulares de suas patentes são Fractal Industry que pertence à empresa Fractal Holdings, e Distributed Energy Management (CRABTREE; JIA, 2009; CRABTREE; SELLERS, 2017; CRABTREE; SELLERS, 2018). 
Existem quatro grupos de cotitulares. Um deles é transnacional e reúne empresas Dell (Dell Marketing, Dell Systems, Dell, Dell Software, Dell Internacional) com Aventail, Credant Technologies, EMC IP Holding, Force 10 Netwaorks, e ASAP Software rexpress, e desenvolveram juntos um sistema de identificação de padrões de fraudes (ZASLAVSKY et al., 2013). Outro consiste numa colaboração chinesa entre a Economy Research Institute Of State Grid Zhejiang Electric Power, Hangzhou Yihe Electric Power Technology Information, State Grid Corporation Of China (SGCC), State Grid Zhejiang Electric Power sobre método de gestão de custo de energia e está protocolada na China (2016) e nos EUA (2017) (LIU et al., 2016).

Uma cotitularidade entre a Highbridge International e a Aastra desenvolveu um mecanismo para otimizar processos de licitação usados para selecionar recursos e serviços.

Jeffrey e Asset Trust desenvolveram juntos métodos automáticos e sistemas para identificar, medir e melhorar categorias de valor para uma cadeia de valor (JEFFREY, 2004b).

\section{Considerações Finais}

Dentro do escopo da pesquisa, as patentes apresentam alta concentração de patentes vivas, com sinais de que essa tecnologia é uma tecnologia emergente. Tal perspectiva é reforçada pela existência de maior concentração das patentes nos anos de 2016 e 2017.

Quanto às primeiras prioridades, elas demonstram notória superioridade de registros realizados na China e nos EUA, além de encontrarem registros em países não produtores da tecnologia, como Senegal, Hong Kong, México, Argentina, Brasil, Itália e Polônia. Tais países são, assim, identificados como mercados potências de exportação da técnica.

Quanto à aplicação de técnicas, a identificação de outliers e a realização de feature selection e clustering (agrupamento), houve apenas identificação de uma patente que tratasse do processo de remoção de ruídos.

Atualmente, não existe patente alguma em nome de pessoas físicas, sendo todas apropriadas ou licenciadas por empresas. As empresas que atuam como maiores players nesse campo são a Alibaba Holding e Fujitso. O titular mais citado foi Jeffrey Edider e o invento mais citado foi Jason Crabtree, cujo titular das patentes é a Fractal Industry.

Embora o domínio dos direitos seja de empresas, as titulares não possuem a atividade financeira como atividade-fim, mas são grandes produtoras de tecnologia. Tal fato deverá ser investigado em futuras pesquisas, podendo ser resultado de esforço de manter segredos de negócios por parte das empresas que atuam em finanças como atividade-fim.

Por fim, o mapeamento demonstrou que a tecnologia está em desenvolvimento, tendo encontrado um salto produtivo em 2016, com avanço do acesso às informações, da maior capacidade de processamento e do uso de tecnologias "nas nuvens". Isso mostrou que haverá espaço nos próximos anos para amadurecimento da tecnologia de aplicação de machine learning ao universo financeiro. 


\section{Referências}

CAVALCANTE, Rodolfo C. et al. Computational Intelligence and Financial Markets: a Survey and Future Directions. Expert Systems with Applications, [S.l.], v. 55, p. 194-211, 2016.

CHAKRABORTY, C.; JOSEPH, A. Machine learning at central banks. Staff Working Paper, [S.l.], n. 674, Bank of England, 2017.

CRABTREE, J.; JIA, J. Inventores. Distributed Energy Management. Titular. Comfort-driven optimization of electric grid utilization. Patente US2010217550. 2009.

CRABTREE, J.; SELLERS, A. Inventores. Fractal Industry. Titular. Quantification for investment vehicle management employing an advanced decision platform. Patente US2017012465. 2017.

CRABTREE, J.; SELLERS, A. Inventores. Fractal Industry. Titular. Quantification for investment vehicle management and insurance process management. Patente WO201885756. 2018.

JEFFREY, E. S. Inventor. Jeffrey E. Titular. A variable group modeling system. Patente US2004088239. 2004a.

JEFFREY, E. S. Inventor. Jeffrey E. Titular. Automated method of and system for identifying, measuring and enhancing categories of value for a value chain. Patente US2004210509. $2004 b$.

JEFFREY, E. S. Inventor. Jeffrey E. Titular. Extended management system. Patente US2009043637. 2009a.

JEFFREY, E. S. Inventor. Jeffrey E. Titular. Market value matrix. Patente US2009018891. 2009a.

JEFFREY, E. S. Inventor. Jeffrey E. Titular. Predictive model development system applied to organization management. Patente US20160239919. 2016.

KHARGONEKAR, P. P.; DAHLEH, M. A. Advancing systems and control research in the era of ML and AI. Annual Reviews in Control, [S.l.], v. 45, p. 1-4, 2018. Disponível em: <https://doi. org/10.1016/j.arcontrol.2018.04.001>. Acesso em: 15 out. 2018.

LIU, W. et al. Inventores. Economy Research Institute Of State Grid Zhejiang Electric Power, Hangzhou Yihe Electric Power Technology Information, State Grid Corporation Of China (SGCC), State Grid Zhejiang Electric Power. Titulares. Management method of power engineering cost. Patente CN105930931. 2016.

ZASLAVSKY, A. et al. Inventores. Asap Software Express, Aventail, Credant Technologies, Dell, Dell International, Dell Marketing, Dell Software, Dell Systems, Emc Ip Holding, Force10 Networks, Maginatics, Mozy, Scaleio, Spanning Cloud Apps. Titulares. Visualization of fraud patterns. Patente US9330416. 2013. 


\section{Sobre os autores}

\section{Vitor M. Quintella}

E-mail: vitor.quintella@gmail.com

Doutorando em Engenharia Industrial pela Universidade Federal da Bahia (2017). Mestre em Engenharia Industrial pela Universidade Federal da Bahia (2017). Graduado em Engenharia Química pela Universidade Federal da Bahia (2013).

Endereço profissional: Universidade Federal da Bahia, Programa de Engenharia Industrial, Escola Politécnica. Rua Aristides Novis, n. 2, Federação, Salvador, BA. CEP: 40210-630.

\section{Cristina M. Quintella}

E-mail: cris5000tina@gmail.com

Doutora interdisciplinar em Ciências Moleculares (University of Sussex, UK). Mestre em Físico-Química pelo Instituto de Química da Universidade Federal do Rio de Janeiro.

Endereço profissional: Universidade Federal da Bahia, Instituto de Química, Campus de Ondina, Ondina, Salvador, BA. CEP: 40170-290.

\section{Antônio Francisco A. Silva Junior}

E-mail: afranc@gmail.com

Pós-doutor como Pesquisador visitante na New York University 2015-2016 (Finance and Risk Engineering Department). Doutor em Engenharia Aeronáutica e Mecânica, em 2006, pelo departamento de Engenharia d e Produção, do Instituto Tecnológico de Aeronáutica (ITA).

Endereço profissional: Escola de Administração da UFBA. Av. Reitor Miguel Calmon, s/n, Vale do Canela, Salvador, BA. CEP: 40110-903.

\section{Cristiano de Oliveira Hora Fontes}

E-mail: cfontes@ufba.br

Doutor em Engenharia Química pela Universidade Estadual de Campinas. Mestre em Engenharia Química pela Universidade Federal da Bahia.

Endereço profissional: Universidade Federal da Bahia, Programa de Engenharia Industrial, Escola Politécnica. Rua Aristides Novis, n. 2, Federação, Salvador, BA. CEP: 40210-630. 